Individual differences in the co-use of reappraisal and emotion suppression in daily life: Implications for daily positive and negative affect

\author{
Baljinder K. Sahdra ${ }^{1}$, Robert Brockman ${ }^{1}$, Steven C. Hayes ${ }^{2}$, Stefan Hofmann ${ }^{3}$, Todd \\ $\operatorname{Kashdan}^{4}$, \& Joseph Ciarrochi ${ }^{1}$ \\ ${ }^{1}$ Australian Catholic University, AUSTRALIA \\ ${ }^{2}$ University of Nevada, USA \\ ${ }^{3}$ Boston University, USA \\ ${ }^{4}$ George Mason University, USA
}

\begin{abstract}
Author Note
Correspondence concerning this article should be addressed to Baljinder K. Sahdra, Institute for Positive Psychology and Education, Australian Catholic University, PO Box 968, North Sydney, NSW 2059. E-mail: baljinder.sahdra@acu.edu.au.

Emails of other authors: rob.brockman@acu.edu.au; stevenchayes@gmail.com; shofmann@bu.edu; kashdan@gmail.com; and joseph.ciarrochi@acu.edu.au.
\end{abstract}




\title{
Individual differences in the co-use of reappraisal and emotion suppression in daily life: Implications for daily positive and negative affect
}

\begin{abstract}
High reappraisal and low suppression are generally seen as desirable outcomes of therapy, but this combination may not benefit those who typically use reappraisal and suppression together. A daily diary study $(N=187 ;$ Mage $=23.9 ; 71 \%$ females; 3,852 days; $M=20.59$ days/person) showed that the group-level correlation between reappraisal and suppression was positive $(r=.32)$, but the within-person correlations varied substantially $(-0.78$ to 0.94$)$. When multiple strategies users employed reappraisal without suppression on a given day, their affect was worse than if they were using no strategy. When single strategy users employed reappraisal with suppression on a given day, their affect was worse than when they used no strategy. Clinicians need to consider how clients co-use strategies in daily life.
\end{abstract}

Keywords: reappraisal; suppression; affect; daily diary; emotion regulation 


\section{Introduction}

High use of cognitive reappraisal (thinking differently about a situation) and low use of suppression (trying not to think about a situation) are generally associated with good mental health (Nezlek \& Kuppens, 2008; Gross, 2015). Reappraisal is a core feature of the cognitive restructuring work in cognitive and behavior therapy (CBT; Beck, 1993), and suppression is a key targeted in acceptance and commitment therapy (ACT; Hayes, 2019). Notwithstanding the debates regarding the similarities and differences between $\mathrm{CBT}$ and ACT, emotional avoidance is generally regarded as unhealthy, and high reappraisal and reduced suppression as desirable outcomes for clients (Arch \& Craske, 2008; Heimberg \& Ritter, 2008). However, is reappraisal universally adaptive and suppression universally maladaptive?

Individually relevant conclusions about psychological processes can be unreliable if they are based on group averages (Hayes et al., 2019). Further, clinical benefits observed at the group level may not be seen within individuals over time (Vilardaga et al., 2013). There is evidence that reappraisal may be helpful for some but not others (Brockman et al., 2017), or may be helpful in some contexts (uncontrollable situations) but unhelpful in others (controllable contexts; Haines et al, 2016). Whilst there is a plethora of research on the benefits of reappraisal and a similarly prolific research on the ills of suppression, there is a dearth of research on the extent to which reappraisal and suppression might be used together by individuals in their daily lives, and how such co-use might impact wellbeing.

The current study employs a daily diary design to test whether people differed in the extent to which they used reappraisal and suppression together on a given day. Given that reappraisal and suppression tend to be only modestly correlated (Brockman et al., 2017), we expected to find a wide range of within-person correlations between daily use of reappraisal and 
suppression. Some people are expected to have a general tendency to use reappraisal and suppression together on a given day ("multiple strategy users"), while others to use one strategy but not the other ("single strategy users"). If such individual differences exist, then the key question becomes: does the pattern of emotion regulation strategy — use of a single strategy vs. co-use of both strategies — make any difference to people's wellbeing in daily life?

To examine this question, we assessed whether the within-person association between reappraisal and suppression added value above the two individual variables in predicting daily positive and negative affect. This allowed us to test the boundary conditions of the 'ideal' configuration of high reappraisal and low suppression. We expected that the extent to which this combination is conducive for high positive and low negative daily affect would depend on whether or not people deviate from their typical pattern of strategy use. Specifically, single strategy users might not do as well on days when they end up using both strategies, relative to the days when they use reappraisal without suppression. However, individuals who typically couse reappraisal and suppression might have the best affect on days when they co-use the two strategies than on days when they use reappraisal without suppression.

\section{Method}

Data were collected from 187 university students (40 men, 133 women, 14 with missing data) with a mean age of 23.9 years $(S D=9.06)$ and a racial composition of $53.1 \%$ Caucasian, 11.7\% Latino/Hispanic, 11.2\% Asian, 7.1\% African-American, 1.6\% Middle Eastern, 1.1\% Native American, and 6.5\% other. The 187 participants provided 3,852 days of data at an average of 20.59 days per person. Pervious analyses of these data have been published elsewhere (Brockman et al., 2017). The current study involves new analysis of the data to test new hypotheses detailed above. 


\section{Measures}

Suppression was measured using the following items adapted from the Emotion Regulation Questionnaire (ERQ; Gross \& John, 2003): “I keep my emotions to myself” (item 2), "When I am feeling positive emotions, I am careful not to express them" (item 4), and "When I am feeling negative emotions, I make sure not to express them” (item 9). Reappraisal was measured using the following items adapted from the ERQ: "When I want to feel more positive emotion (such as joy or amusement), I change what I'm thinking about", and "When I want to feel less negative emotion (such as sadness or anger), I change what I'm thinking about". Daily Positive and Negative Affect were measured using six positively valanced adjectives (excited, enthusiastic, happy, relaxed, calm, and satisfied) and six negatively valanced adjectives (nervous, embarrassed, upset, sad, bored, and disappointed), respectively. Participants responded using a 7point scale ( 1 = "Did not feel this way at all"; 7 = "Felt this way very strongly"). The reliabilities of all measures (as estimated by ICCs) were above .90 (see details in Table S1 of Online Supplementary Materials).

\section{Results}

The means, standard deviations, reliabilities and correlations between the study variables are reported in Table S1. Not surprisingly, negative and positive affect were negatively correlated $(r=-.36, p<.001)$. Negative affect was unrelated to reappraisal $(r=.01, p>.20)$ but positively related to suppression $(r=.21, p<.001)$, whereas positive affect was positively related to reappraisal $(r=.34, p<.001)$ and negatively linked to suppression $(r=-.17, p<.05)$. These correlations are consistent with the general understanding that reappraisal tends to be adaptive and suppression maladaptive for well-being (Gross, 2015). Table S1 also reports a moderate positive correlation between reappraisal and suppression $(r=.32, p<.001)$. 
To examine how consistent or "fixed" the association between reappraisal and suppression was between study participants, we examined the link between the two variables in a multilevel model with days nested within individuals. The first model had varying intercepts but the slope of the link between reappraisal and suppression were fixed across participants. The second model, which allowed both the intercepts and slopes to vary across participants, fit the data better as per a $\log$ likelihood test $\left(X^{2}(2)=102.28, p<.001\right)$. See Table S2 for a full set of fit statistics comparing the two models. Figure S1 in online supplementary materials shows the multilevel model-implied trajectories of daily reappraisal linked with daily suppression for individual participants. For some people, reappraisal was negatively linked with suppression over the course of the study period, while for others the two variables were unrelated or positively linked.

We next computed within-person correlations between daily reappraisal and suppression, which ranged from -0.78 to 0.94 . Figure $S 2$ in online supplementary materials compares the distribution of the correlations with a theoretical normal distribution, showing evidence of normality in our sample. That is, people reliably differed in the extent to which they use a single strategy (negative correlation) or both strategies (positive correlation) in daily life.

Finally, we examined whether or not the correlation between daily reappraisal and suppression added value over and above the two individual variables in predicting daily positive and negative affect. Specifically, we tested whether the interaction between reappraisal and suppression for predicting positive or negative affect was moderated by the extent to which reappraisal and suppression were correlated within persons across days. The 3-way interaction of daily reappraisal, daily suppression and the within-person correlation between the two variables predicting positive and negative affect was tested in multilevel models with random intercepts. 
Table 1 contains the fixed effects estimates and 95\% confidence intervals (CIs). The CIs for the 3-way interaction effects in both models were reasonably narrow and did not include zero.

Figure 1 visually depicts the 3-way interaction effects at -/+ 2 SD levels. To see additional levels, see Figures S3 to S6 in Online Supplementary Materials for the 3-way interaction effect in unstandardized units of all variables, including rug plots showing the distribution of the variable at the x-axis, which is fairly evenly distributed in all panels.

When daily suppression was low (panels A \& C of Figure 1), reappraisal was positively linked to healthier daily affect profiles (high positive affect and low negative affect) for single strategy users, but negatively linked among those that normally use both strategies. When daily suppression was high (B \& D), reappraisal was unrelated to affect among single strategy users and positively related to affect among those who use both strategies. Among both-strategies users, reappraisal was beneficial for affect on days when they also used daily suppression (B \& D), but potentially harmful on days when they only used the single strategy of reappraisal (A \& C). Among single strategy users, reappraisal was beneficial for affect on days when they only used this strategy and not suppression (A \& C), but neutral on days they attempted to use both strategies (B \& D).

\section{Discussion}

In a daily diary study, we found that reappraisal was not universally adaptive or suppression universally maladaptive. These strategies were beneficial, neutral or harmful, depending on individuals' general tendency to co-use the strategies and the specific context of using a single strategy or both strategies on a given day. When multiple strategies users employ reappraisal without suppression on a given day, their affect is worse than if they were using no 
strategy. Encouraging an exclusive use of reappraisal in these people might be counterproductive. However, the opposite conclusion would be made for single strategy users. When they use reappraisal with suppression on a given day, their affect is worse than when they use no strategy. These people appear better off using either no strategy at all or both strategies together.

The study has several limitations. We relied exclusively on self-reports. Future research would benefit from a combination of self-reports and therapist-reports. Further, the participants in our study were from the general population of an English-speaking culture. It remains to be seen whether we will observe a similar pattern of results in clinical samples, or in other cultures.

Nevertheless, the results are consistent with a growing recognition in psychology that clinical benefits seen at the group level may not apply to specific individuals at any given time or within individuals over time (Hayes et al., 2019). They highlight the importance of understanding how clients' default emotion regulation strategies operate in their day-to-day lives. Generally speaking, cognitive restructuring in therapy need not depend on suppression. But our results suggest that clinicians might benefit from paying attention to the potential co-use of reappraisal and suppression, at least in some clients. In particular, clinicians might ask: on days when a client co-uses reappraisal and suppression, and experiences high wellbeing, what did that co-use involve for this particular client on that particular day, and how might those behaviors be facilitated through therapy. In this manner, daily assessments of clients' emotion regulation may facilitate personalized and precision treatments. 


\section{References}

Arch, J. J., \& Craske, M. G. (2008). Acceptance and commitment therapy and cognitive behavioral therapy for anxiety disorders: Different treatments, similar mechanisms? Clinical Psychology: Science and Practice, 15(4), 263-279.

Beck, A. T. (1993). Cognitive therapy: past, present, and future. Journal of Consulting and Clinical Psychology, 61(2), 194-198.

Brockman, R., Ciarrochi, J., Parker, P. \& Kashdan, T. (2017). Emotion regulation strategies in daily life: mindfulness, cognitive reappraisal and emotion suppression. Cognitive Behaviour Therapy, 46, 91-113. doi: 10.1080/16506073.2016.1218926

Gross, J. J. (2015). Emotion regulation: Current status and future prospects. Psychological Inquiry, 26(1), 1-26.

Gross, J. J., \& John, O. P. (2003). Individual differences in two emotion regulation processes: Implications for affect, relationships, and well-being. Journal of Personality and Social Psychology, 85, 348-362. doi:10.1037/0022-3514.85.2.348

Haines, S. J., Gleeson, J., Kuppens, P., Hollenstein, T., Ciarrochi, J., Labuschagne, I. Grace, C., Koval, P. (2016). The Wisdom to Know the Difference. Psychological Science, 27(12), 1651-1659.

Hayes, S. (2019). A Liberated Mind: The essential guide to ACT. Random House. 
Hayes, S. C., Hofmann, S. G., Stanton, C. E., Carpenter, J. K., Sanford, B. T., Curtiss, J. E., \& Ciarrochi, J. (2019). The role of the individual in the coming era of process-based therapy. Behaviour Research and Therapy, 117, 40-53.

Heimberg, R. G., \& Ritter, M. R. (2008). Cognitive behavioral therapy and acceptance and commitment therapy for the anxiety disorders: Two approaches with much to offer. Clinical Psychology: Science and Practice, 15(4), 296-298.

Nezlek, J. B., \& Kuppens, P. (2008). Regulating positive and negative emotions in daily life. Journal of Personality, 76(3), 561-580.

Vilardaga, R., Hayes, S. C., Atkins, D. C., Bresee, C. \& Kambiz, A. (2013). Comparing experiential acceptance and cognitive reappraisal as predictors of functional outcome among individuals with psychotic symptoms. Behaviour Research and Therapy, 51, 425-433. doi: 10.1016/j.brat.2013.04.003 
Table 1. Fixed effects and 95\% confidence intervals from multilevel models

\begin{tabular}{|c|c|c|c|c|c|c|c|c|}
\hline & \multicolumn{4}{|c|}{ Positive Affect } & \multicolumn{4}{|c|}{ Negative Affect } \\
\hline & Estimate & $95 \%$ & & & Estimate & & $\% \mathrm{CI}$ & \\
\hline Intercept & -0.06 & {$[-0.15$} & $0.03]$ & & 0.09 & {$[-0.01$} & $0.17]$ & \\
\hline $\mathrm{C}$ & -0.01 & {$[-0.10$} & $0.08]$ & & -0.05 & {$[-0.14$} & $0.03]$ & \\
\hline $\mathrm{R}$ & 0.21 & {$[0.17$} & $0.26]$ & $* * *$ & -0.05 & {$[-0.10$} & $0.00]$ & \\
\hline S & -0.10 & {$[-0.14$} & $-0.06]$ & $* * *$ & 0.17 & {$[0.13$} & $0.21]$ & $* * *$ \\
\hline $\mathrm{C} \times \mathrm{R}$ & -0.12 & {$[-0.16$} & $-0.07]$ & $* * *$ & 0.12 & {$[0.07$} & $0.17]$ & $* * *$ \\
\hline $\mathrm{C} \times \mathrm{S}$ & 0.07 & {$[0.03$} & $0.11]$ & $* * *$ & -0.04 & {$[-0.08$} & $0.00]$ & \\
\hline $\mathrm{R} \times \mathrm{S}$ & 0.00 & {$[-0.04$} & $0.03]$ & & -0.04 & {$[-0.07$} & $0.00]$ & \\
\hline $\mathrm{C} \times \mathrm{R} \times \mathrm{S}$ & 0.05 & {$[0.03$} & $0.08]$ & $* * *$ & -0.06 & {$[-0.09$} & $-0.03]$ & $* * *$ \\
\hline
\end{tabular}

Note. C: Co-use of daily reappraisal and suppression; R: Daily reappraisal; S: Daily suppression; $* * * p<.001$ 

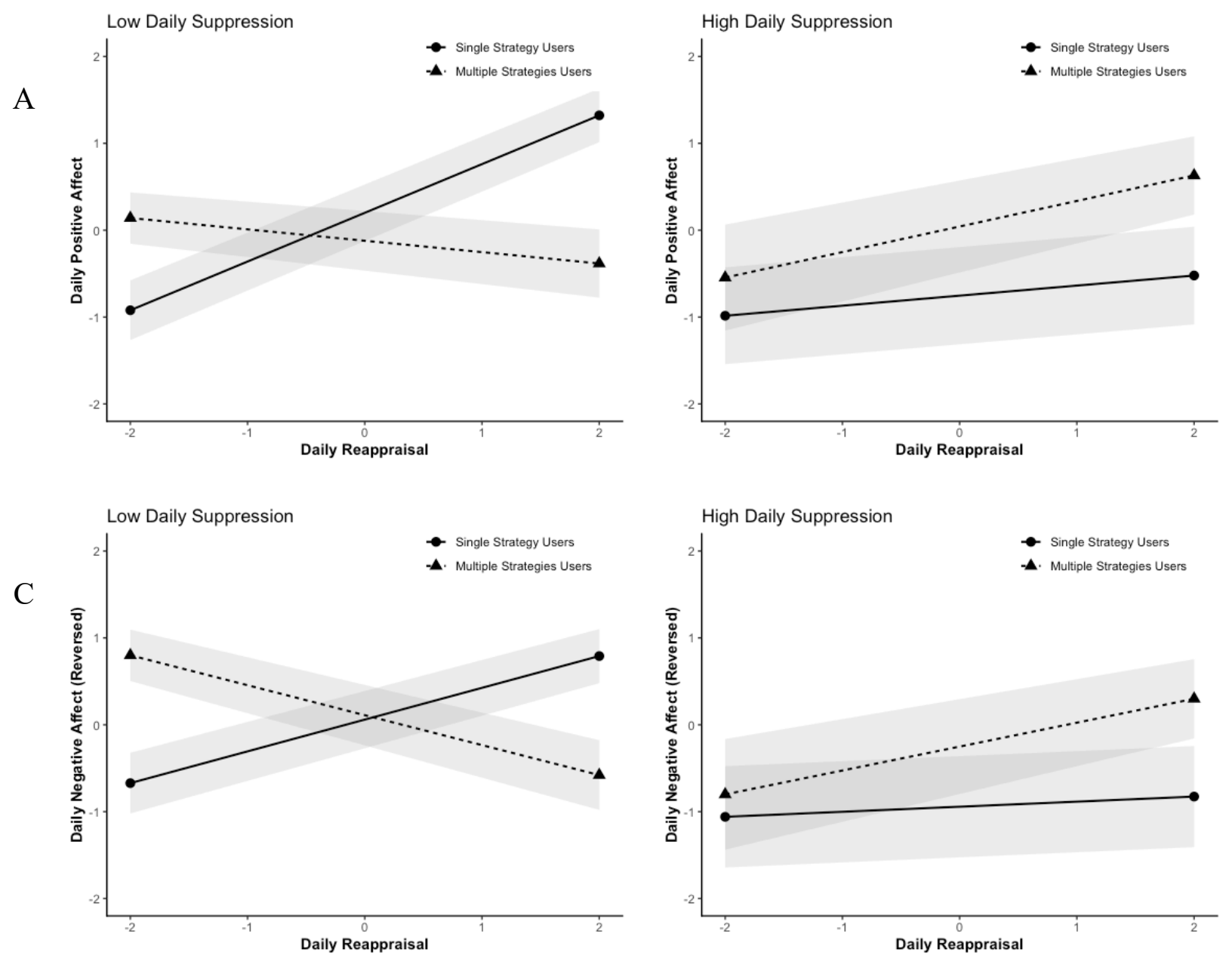

Figure 1. Three-way interactions of daily reappraisal, daily suppression and their correlation predicting daily positive affect (A \& B) and reversed-scored negative affect (C \& D). Single strategy users have a negative correlation and multiple strategy users have a positive correlation between daily reappraisal and suppression. 


\section{Supplementary Materials}

\section{Tables}

Table S1. Means, standard deviations, reliabilities (as estimated by ICCs) and inter-correlations of the study variables

\begin{tabular}{lccccc}
\hline Measure & $M(S D)$ ICC & 1 & 2 & 3 & 4 \\
\hline 1) Negative Affect & $9.84(4.77) .90$ & - & $-.36^{* *}$ & -.01 & $.21^{* *}$ \\
2) Positive Affect & $16.38(5.84) .92$ & - & $.34^{* *}$ & $-.17^{*}$ \\
3) Reappraisal & $7.40(3.43) .97$ & & - & $.32^{* *}$ \\
4) Suppression & $8.13(4.04) .96$ & & & - \\
\hline
\end{tabular}

Note. ICC: Intra-Class Correlation. ${ }^{*} p<.05 ;{ }^{*} p<.01$ 
Table S2. Comparison of two multilevel models of reappraisal linked to suppression

\begin{tabular}{lrrrrrrrrr}
\hline & $d f$ & AIC & BIC & logLik & Deviance & $\chi^{2}$ & $d f$ & $p$ \\
\hline Model 1 & 4 & 7044.55 & 7068.87 & -3518.27 & 7036.55 & - & - & - \\
& & & & & & & & \\
Model 2 & 6 & 6946.27 & 6982.75 & -3467.13 & 6934.27 & 102.28 & 2 & $<.001$ \\
\hline
\end{tabular}

Note. Model 1: Random intercepts only; Model 2: Random intercepts and slopes; logLik: log likelihood test; AIC: Akaike Information Criteria; BIC: Bayesian Information Criteria 


\section{Figures}

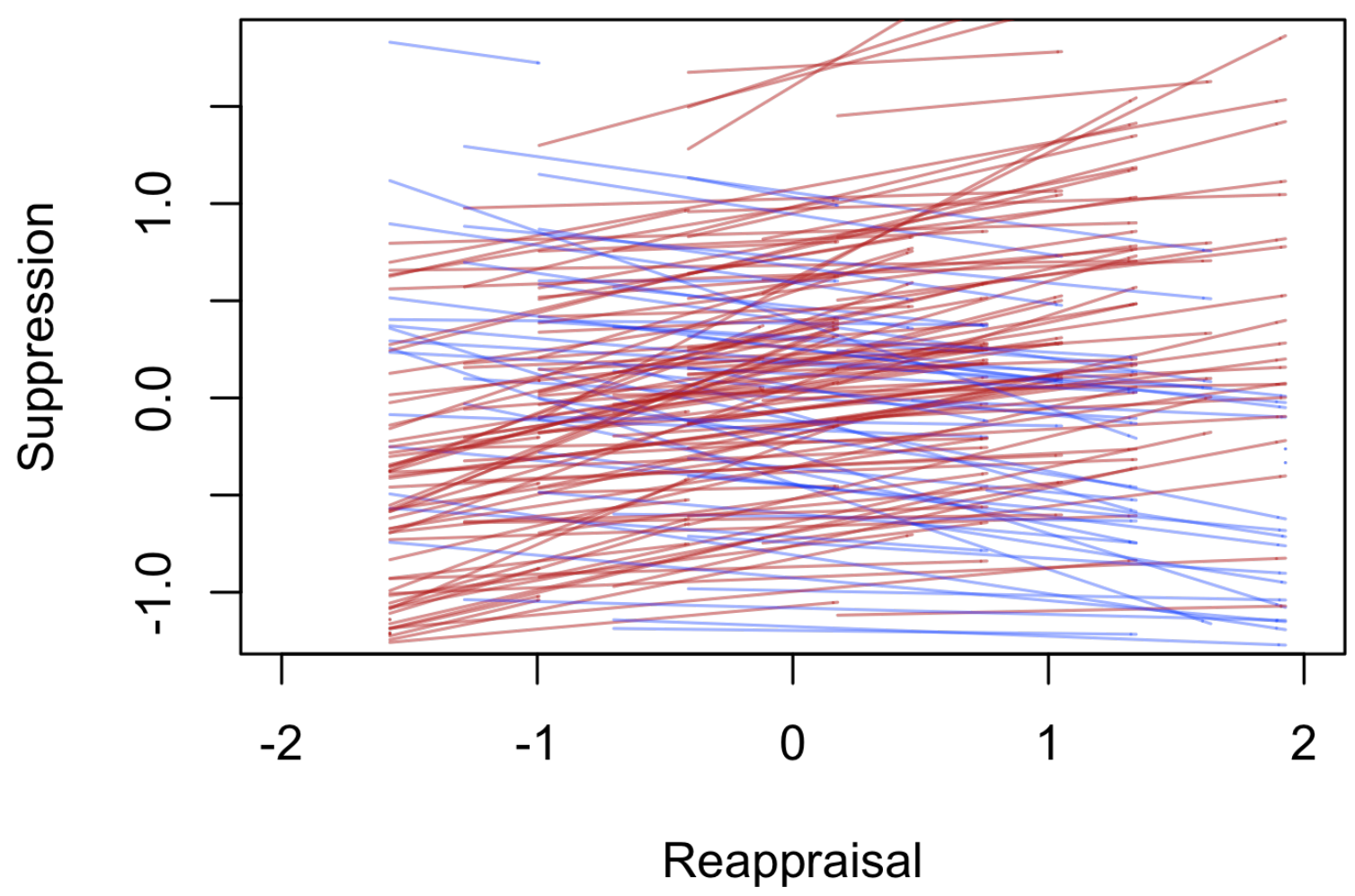

Figure S1: Model implied random intercepts and slopes from a multilevel model linking reappraisal and suppression 

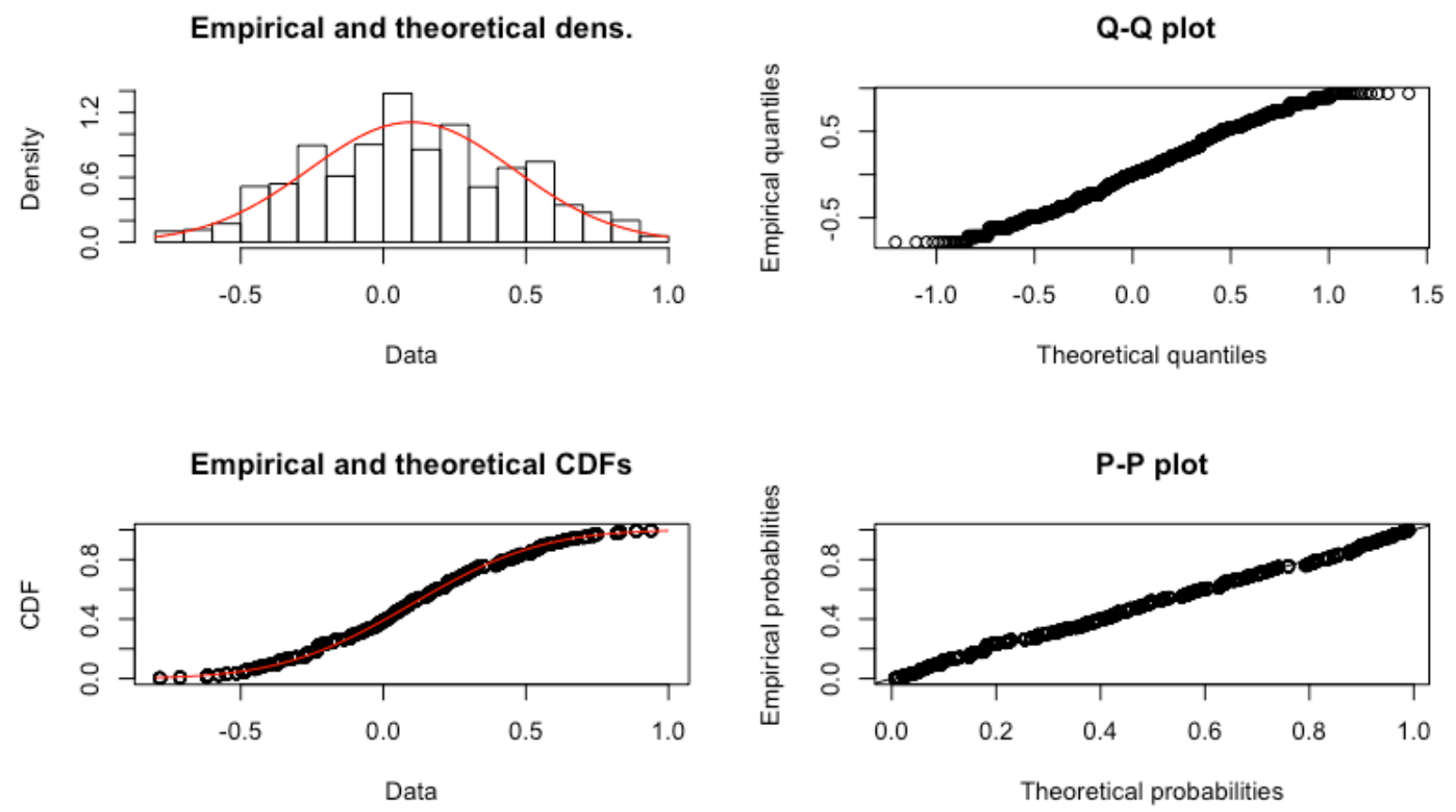

Figure S2: Distribution of the correlations between daily reappraisal and suppression in comparison to the theoretical normal distribution 


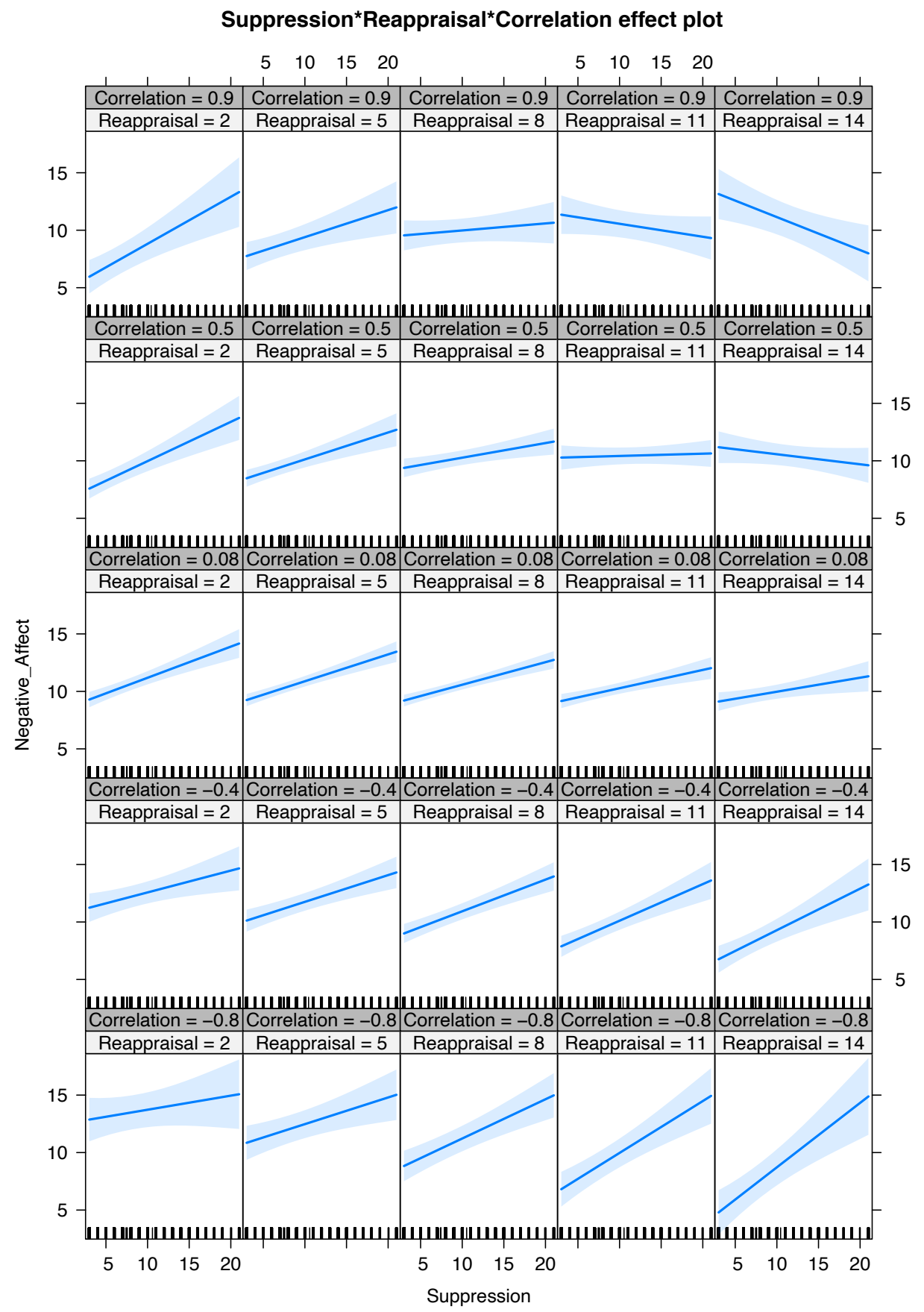

Figure S3: Model implied associations between suppression and negative affect at different

levels of reappraisal and the correlation between suppression and reappraisal. The rug plot shows the distribution of suppression. 


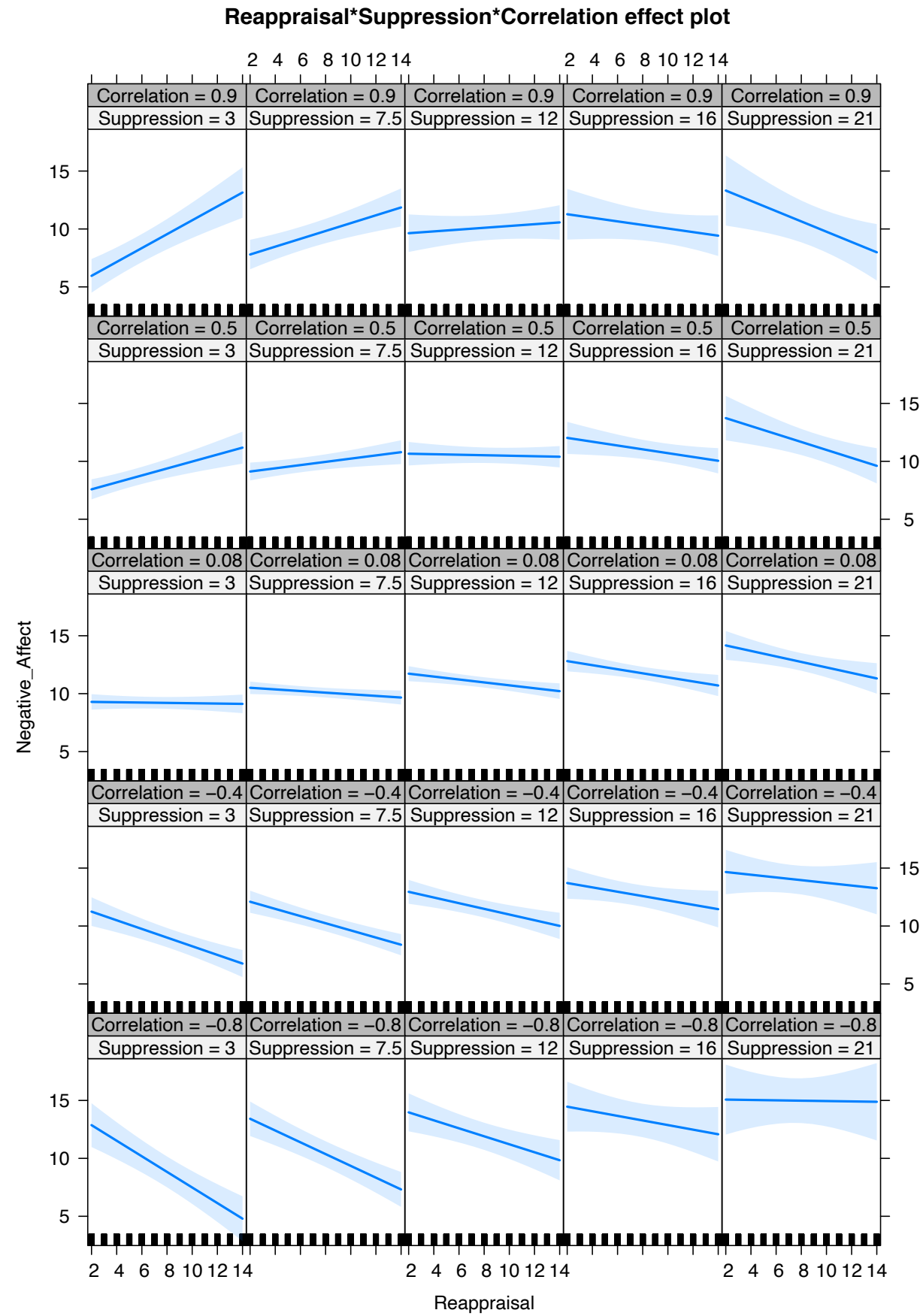

Figure S4: Model implied associations between reappraisal and negative affect at different levels of suppression and the correlation between suppression and reappraisal. The rug plot shows the distribution of reappraisal. 


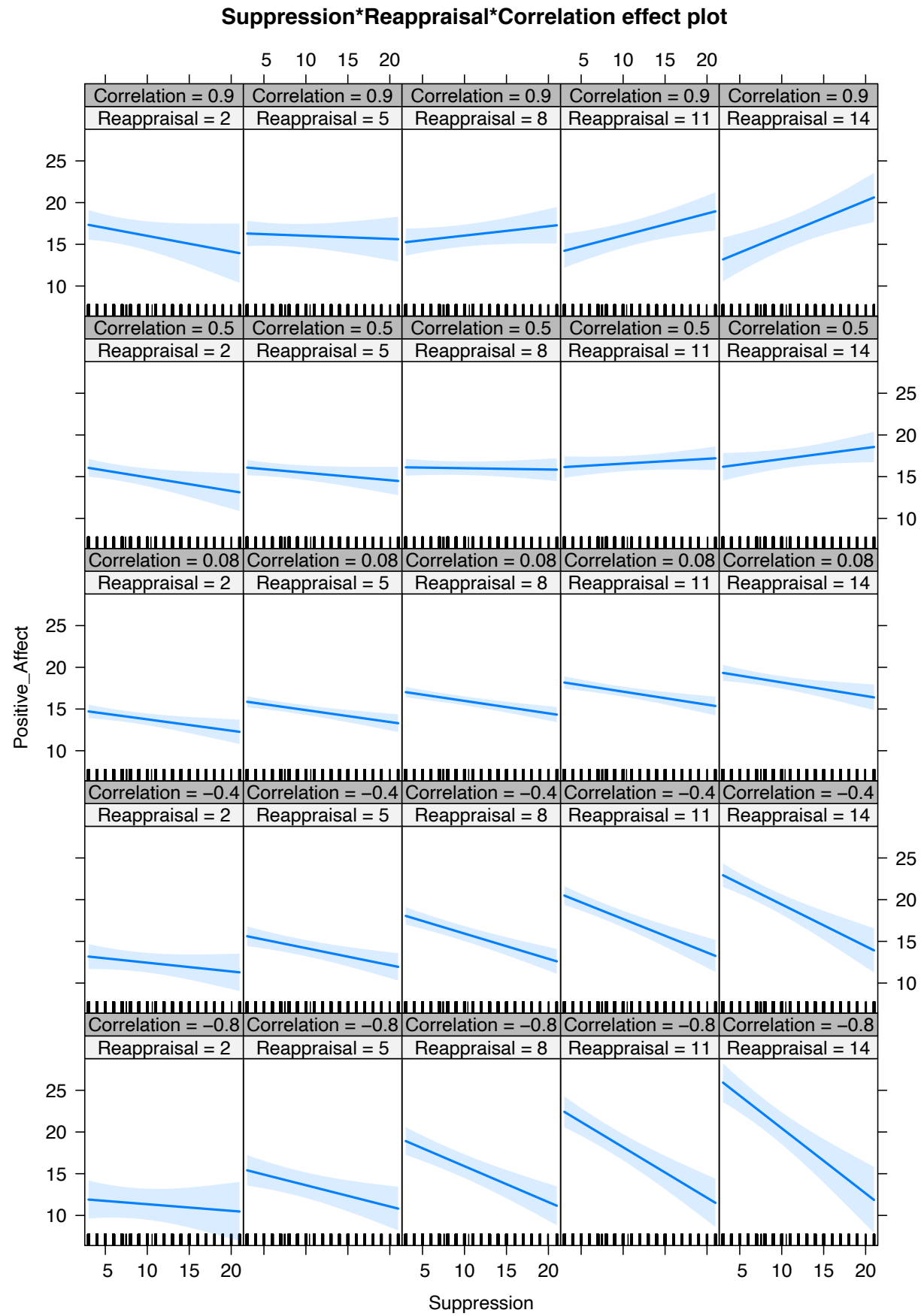

Figure S5: Model implied associations between suppression and positive affect at different levels of reappraisal and the correlation between suppression and reappraisal. The rug plot shows the distribution of suppression. 


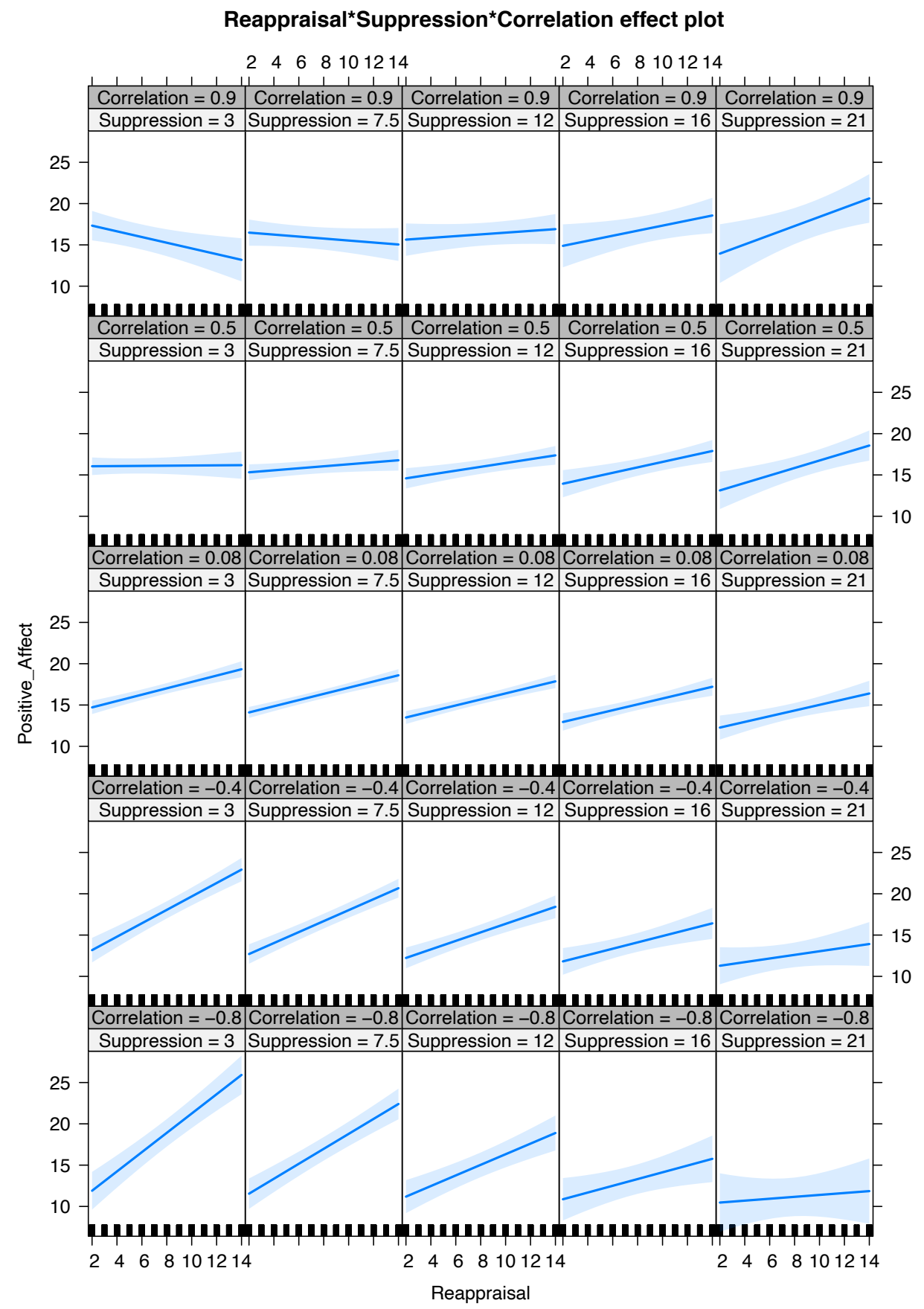

Figure S6: Model implied associations between reappraisal and positive affect at different levels of suppression and the correlation between suppression and reappraisal. The rug plot shows the distribution of reappraisal. 